\title{
On the asymptotic stability of a class of jump-diffusions of neutral type with impulses
}

\author{
Feng Jiang ${ }^{1 *}$, Hua Yang ${ }^{2}$ and Xinquan Zhao'
}

${ }^{*}$ Correspondence:
fjiang78@gmail.com
'School of Statistics \& Mathematics,
Zhongnan University of Economics
and Law, Wuhan, 430073, China
Full list of author information is
available at the end of the article

\begin{abstract}
This paper is concerned with the asymptotic stability in the pth moment for a class of jump-diffusions of neutral type with impulses. Sufficient conditions ensuring the stability of jump-diffusions of neutral type with impulses are established by means of the Banach fixed point theorem. The results obtained here generalize and improve some well-known results.
\end{abstract}

Keywords: impulses; Poisson jump; mild solution; asymptotic stability

\section{Introduction}

Recently, the existence, uniqueness and stability of solutions of stochastic differential equations, especially stochastic partial differential equations [1-5], have been considered by many authors [6]. Besides stochastic effects, impulsive effects also occur in real systems. The study of impulsive systems in a separable Hilbert space is motivated by modeling some evolution phenomena arising in physics, communications, engineering, etc. [7-9].

In addition, many dynamical systems not only depend on present and past states, but also involve derivative with delays, and neutral systems are often used to describe such systems. It should pointed out that there are a few works about the existence and stability of mild solutions of neutral systems [10-16]. Meanwhile, there are also a few works on jump diffusions, and some results on the existence, uniqueness, stability and qualitative properties of solutions have been obtained. For example, Bao et al. [17] studied almost sure asymptotic stability of stochastic partial differential equations with jumps. Bao et al. [18] discussed stability in distribution of mild solutions to stochastic partial differential equations with jumps. Cui et al. [19] discussed exponential stability for neutral stochastic partial differential equations with delays and Poisson jumps. Peszat and Zabczyk [20] discussed the theory of stochastic partial differential equations with Lévy noise. Motivated by the above papers, in the paper we aim to study the existence and asymptotic stability of a class of jump-diffusions of neutral type with impulses by means of the Banach fixed point theorem, the results obtained here generalize the main results from Mahmudov [10], Jiang and Shen [14], Sakthivel [21].

The organization of the paper is as follows. In the next section, we introduce some notations and definitions of mild solution and asymptotic stability. Then we give sufficient conditions ensuring the stability of jump-diffusions of neutral type with impulses by means of the Banach fixed point theorem.

(2013 Jiang et al.; licensee Springer. This is an Open Access article distributed under the terms of the Creative Commons Attribution License (http://creativecommons.org/licenses/by/2.0), which permits unrestricted use, distribution, and reproduction in any medium, provided the original work is properly cited. 


\section{Preliminaries}

Throughout this paper, let $\left(\Omega, \mathcal{F},\left\{\mathcal{F}_{t}\right\}_{t \geq 0}, P\right)$ be a complete probability space with a filtration $\left\{\mathcal{F}_{t}\right\}_{t \geq 0}$ satisfying the usual conditions (i.e., it is increasing and right-continuous while $\mathcal{F}_{0}$ contains all $P$-null sets) [6]. Moreover, let $X, Y$ be two real separable Hilbert spaces, and let $L(Y, X)$ denote the space of all bounded linear operators from $Y$ into $X$.

For simplicity, we use the notation $|\cdot|$ to denote the norm in $X, Y$ and $\|\cdot\|$ to denote the operator norm in $L(X, X)$ and $L(Y, X)$. Let $\langle\cdot\rangle_{X},\langle\cdot\rangle_{Y}$ denote the inner products of $X, Y$, respectively. Let $\{w(t): t \geq 0\}$ denote an $Y$-valued Wiener process defined on the probability space $\left(\Omega, \mathcal{F},\left\{\mathcal{F}_{t}\right\}_{t \geq 0}, P\right)$ with the covariance operator $Q$, that is, $E\langle w(t), x\rangle_{Y}\langle w(s), y\rangle_{Y}=$ $(t \wedge s)\langle Q x, y\rangle_{Y}$ for all $x, y \in Y$, where $Q$ is a positive, self-adjoint, trace class operator on $Y$. In particular, we denote by $w(t)$ a $Y$-valued $Q$-Wiener process with respect to $\left\{\mathcal{F}_{t}\right\}_{t \geq 0}$. We assume that there exists a complete orthonormal system $\left\{e_{i}\right\}$ in $Y$, a bounded sequence of nonnegative real numbers $\lambda_{i}$ such that $Q e_{i}=\lambda_{i} e_{i}, i=1,2, \ldots$, and a sequence $\left\{\beta_{i}\right\}_{i \geq 1}$ of independent Brownian motions such that $\langle w(t), e\rangle=\sum_{i=1}^{\infty} \sqrt{\lambda_{i}}\left\langle e_{i}, e\right\rangle \beta_{i}(t), e \in Y$, and $\mathcal{F}_{t}=\mathcal{F}_{t}^{w}$, where $\mathcal{F}_{t}^{w}$ is the $\sigma$-algebra generated by $\{w(s): 0 \leq s \leq t\}$. Let $L_{2}^{0}=L_{2}\left(Q^{1 / 2} Y ; X\right)$ be the space of all Hilbert-Schmidt operators from $Q^{1 / 2} Y$ to $X$ with the inner product $\langle u, \xi\rangle_{L_{2}^{0}}=\operatorname{tr}[u Q \xi]$; see, for example, [8].

Let $p=(p(t)), t \in D_{p}$ be a stationary $\mathcal{F}_{t}$-Poisson point process with characteristic measure $\lambda$. Denote by $N(d t, d v)$ the Poisson counting measure associated with $p$, that is, $N(t, \mathcal{Z})=\sum_{s \in D_{p}, s \leq t} I_{\mathcal{Z}}(p(s))$ with a measurable set $\mathcal{Z} \in \mathcal{B}(Y-\{0\})$, which denotes the Borel $\sigma$-field of $Y-\{0\}$. Let $\widetilde{N}(d t, d v)=N(d t, d v)-d t \lambda(d v)$ be the compensated Poisson measure, which is independent of $w(t)$. Denote by $\mathcal{P}^{2}([0, T] \times \mathcal{Z} ; X)$ the space of all predictable mappings $L:[0, T] \times \mathcal{Z} \times \Omega \rightarrow X$ for which

$$
\int_{0}^{T} \int_{\mathcal{Z}} E|L(t, v)|^{2} d t \lambda(d v)<\infty
$$

We may then define the $X$-valued stochastic integral $\int_{0}^{T} \int_{\mathcal{Z}} L(t, v) \tilde{N}(d t, d v)$, which is a centered square-integrable martingale [5]. We always assume that $w(t)$ and $\tilde{N}$ are independent of $\mathcal{F}_{0}$.

Now consider a class of jump-diffusions of neutral type with impulses of the form

$$
\left\{\begin{aligned}
d[x(t)-u(t, x(t-\rho(t)))]= & {[A x(t)+f(t, x(t-\tau(t)))] d t } \\
& +g(t, x(t-\delta(t))) d w(t) \\
& +\int_{\mathcal{Z}} h(t, x(t-\kappa(t)), v) \widetilde{N}(d t, d v), \quad t \geq 0, t \neq t_{k}, \\
\triangle x\left(t_{k}\right)=x\left(t_{k}^{+}\right)-x\left(t_{k}^{-}\right)=I_{k}( & \left.x\left(t_{k}^{-}\right)\right), \quad t=t_{k}, k=1,2, \ldots, m,
\end{aligned}\right.
$$

with the initial data $x(t)=\varphi \in C_{\mathcal{F}_{0}}^{b}([-\tau, 0], X)$, where $u: R_{+} \times X \rightarrow X, f: R_{+} \times X \rightarrow X, g$ : $R_{+} \times X \rightarrow L(Y, X), h: R_{+} \times X \times \mathcal{Z} \rightarrow X$ are all Borel measurable; $\rho: R_{+} \rightarrow[0, \tau], \tau: R_{+} \rightarrow$ $[0, \tau], \delta: R_{+} \rightarrow[0, \tau], \kappa: R_{+} \rightarrow[0, \tau]$ are continuous; $A$ is the infinitesimal generator of a semigroup of bounded linear operators $S(t), t \geq 0$, in $X ; I_{k}: X \rightarrow X$. Furthermore, the fixed moments of times $t_{k}$ satisfy $0<t_{1}<\cdots<t_{m}<\lim _{k \rightarrow \infty} t_{k}=\infty, x\left(t_{k}^{+}\right)$and $x\left(t_{k}^{-}\right)$represent the right and left limits of $x(t)$ at $t=t_{k}$, respectively. Also $\Delta x\left(t_{k}\right)=x\left(t_{k}^{+}\right)-x\left(t_{k}^{-}\right)$represents the jump in the state $x$ at time $t_{k}$ with $I_{k}$ determining the size of the jump. $\tau>0$ and $C=$ $C([-\tau, 0] ; X)$ denotes a family of all right-continuous functions with left-hand limits $\eta$ from $[-\tau, 0]$ to $X$. Denote the norm of $\eta(t)$ by $\|\eta\|_{C}=\sup _{t \in[-\tau, 0]} E|\eta(t)|$. Here, $C_{\mathcal{F}_{0}}^{b}([-\tau, 0], X)$ is 
a family of all almost surely bounded, $\mathcal{F}_{0}$-measurable, continuous random variables from $[-\tau, 0]$ to $X$.

Suppose that $\{S(t), t \geq 0\}$ is an analytic semigroup with its infinitesimal generator $A$. For a basic reference, the reader is referred to Pazy [22]. We always assume $0 \in \varrho(A)$, the resolvent set of $-A$. For any $\alpha \in[0,1]$, it is possible to define the fractional power $(-A)^{\alpha}$ which is a closed linear operator with its domain $\mathcal{D}\left((-A)^{\alpha}\right)$.

Definition 1 A process $\{x(t), t \in[0, T]\}, 0 \leq T<\infty$, is called a mild solution of Eq. (1) if

(i) $x(t)$ is adapted to $\mathcal{F}_{t}, t \geq 0$ with $\int_{0}^{T}|x(t)|^{2} d t<\infty$ a.s.;

(ii) $x(t) \in X$ has càdlàg paths on $t \in[0, T]$ a.s. and for each $t \in[0, T], x(t)$ satisfies the integral equation

$$
\begin{aligned}
x(t)= & S(t)[\varphi(0)-u(0, x(-\rho(0)))]+u(t, x(t-\rho(t))) \\
& +\int_{0}^{t} A S(t-s) u(s, x(s-\rho(s))) d s \\
& +\int_{0}^{t} S(t-s) f(s, x(s-\tau(s))) d s \\
& +\int_{0}^{t} S(t-s) g(s, x(s-\delta(s))) d w(s) \\
& +\int_{0}^{t} \int_{\mathcal{Z}} S(t-s) h(s, x(s-\kappa(s), v)) \tilde{N}(d s, d v) \\
& +\sum_{0<t_{k}<t} S\left(t-t_{k}\right) I_{k}\left(x\left(t_{k}^{-}\right)\right),
\end{aligned}
$$

and $\varphi \in C_{\mathcal{F}_{0}}^{b}([-\tau, 0], X)$.

Moreover, for the purposes of stability, we always assume that $u(t, 0)=0, f(t, 0)=0$, $g(t, 0)=0, h(t, 0, v)=0, I_{k}(0)=0(k=1,2, \ldots)$. Hence Eq. (1) has a trivial solution when $\varphi=0$.

Definition 2 Let $p \geq 2$ be an integer. The trivial solution of Eq. (1) or Eq. (1) itself is said to be stable in the $p$ th moment if for arbitrarily given $\varepsilon>0$, there exists $\delta>0$ such that $\|\varphi\|_{C}<\delta$ guarantees that

$$
E\left(\sup _{t \geq 0}|x(t)|^{p}\right)<\varepsilon
$$

Definition 3 Let $p \geq 2$ be an integer. The trivial solution of Eq. (1) or Eq. (1) itself is said to be asymptotically stable (or globally asymptotically stable) in the $p$ th moment if it is stable in the $p$ th moment and for any $\varphi \in C_{\mathcal{F}_{0}}^{b}([-\tau, 0], X)$,

$$
\lim _{T \rightarrow \infty} E\left(\sup _{t \geq T}|x(t)|^{p}\right)=0
$$

When $p=2$, we say Eq. (1) is mean square asymptotically stable (or mean square globally asymptotically stable). 
To establish the stability of Eq. (1), we employ the following assumptions.

(H1) A is the infinitesimal generator of a semigroup of bounded linear operators $S(t)$, $t \geq 0$, in $X$ satisfying $|S(t)| \leq M e^{-a t}, t \geq 0$, for some constants $M \geq 1$ and $0<a \in R_{+}$.

(H2) The functions $f, g$ and $h$ satisfy the following conditions: there exists a constant $K$ such that for any $x, y \in X$ and $t \geq 0$,

$$
\begin{aligned}
& |f(t, x)-f(t, y)|^{2} \leq K|x-y|^{2}, \\
& \|g(t, x)-g(t, y)\|^{2} \leq K|x-y|^{2}, \\
& \int_{\mathcal{Z}}|h(t, x, v)-h(t, y, v)|^{2} \lambda(d v) \leq K|x-y|^{2} .
\end{aligned}
$$

(H3) There exist a number $\alpha \in[0,1]$ and a positive constant $\bar{K}$ such that for any $x, y \in X$ and $t \geq 0, u(t, x) \in \mathcal{D}\left((-A)^{\alpha}\right)$ and

$$
\left|(-A)^{\alpha} u(t, x)-(-A)^{\alpha} u(t, y)\right|^{2} \leq \bar{K}|x-y|^{2} .
$$

(H4) There exists a constant $q_{k}$ such that $\left|I_{k}(x)-I_{k}(y)\right|^{2} \leq q_{k}|x-y|^{2}$ for each $x, y \in X$ $(k=1, \ldots, m)$.

\section{Asymptotic stability}

In this section, we consider the asymptotic stability of Eq. (1) by means of the fixed point theory. Let $H$ be the space of all $\mathcal{F}_{0}$-adapted processes $\psi(t, \bar{w}):[0, \infty) \times \Omega \rightarrow R$ which is almost certainly continuous in $t$ for fixed $\bar{w} \in \Omega$. Moreover, $\psi(s, \bar{w})=\varphi(s)$ for $s \in[-\tau, 0]$ and $E|\psi(t, \bar{w})|^{2} \rightarrow 0$ as $t \rightarrow \infty$.

Now let us state the following well-known lemma [22], which will be used in the sequel in the proof of the main result.

Lemma 1 If $(\mathrm{H} 1)$ holds and $0 \in \varrho(A)$, then for any $\beta \in(0,1]$,

(i) for each $x \in \mathcal{D}\left((-A)^{\beta}\right), S(t)(-A)^{\beta} x=(-A)^{\beta} S(t) x$;

(ii) there exist constants $M_{\beta}>0$ and $a \in R_{+}$such that $\left\|(-A)^{\beta} S(t)\right\| \leq M_{\beta} t^{-\beta} e^{-a t}, t>0$.

We can now state our main result of this paper.

Theorem 1 If (H1)-(H4) hold for some $\alpha \in(1 / 2,1]$, then Eq. (1) is mean square globally asymptotically stable provided

$$
\bar{K}\left|(-A)^{-\alpha}\right|^{2}+\bar{K} M_{1-\alpha}^{2} a^{-2 \alpha} \Gamma(2 \alpha-1)+M^{2} K a^{-2}+K M^{2} a^{-1}+M^{2} \bar{L}<1 / 6,
$$

where $\bar{L}=e^{-2 a T} E\left(\sum_{k=1}^{m}\left|q_{k}\right|\right)$.

Proof Define an operator $\pi: H \rightarrow H$ by $\pi(x)(t)=\Psi(t)$ for $t \in[-\tau, 0]$ and for $t \geq 0$,

$$
\begin{aligned}
(\pi x)(t)= & S(t)[\varphi(0)-u(0, x(-\rho(0)))]+u(t, x(t-\rho(t))) \\
& +\int_{0}^{t} A S(t-s) u(s, x(s-\rho(s))) d s
\end{aligned}
$$




$$
\begin{aligned}
& +\int_{0}^{t} S(t-s) f(s, x(s-\tau(s))) d s+\int_{0}^{t} S(t-s) g(s, x(s-\delta(s))) d w(s) \\
& \quad \times \int_{0}^{t} \int_{\mathcal{Z}} S(t-s) h\left(s, x(s-\kappa(s), v) \widetilde{N}(d s, d v)+\sum_{0<t_{k}<t} S\left(t-t_{k}\right) I_{k}\left(x\left(t_{k}^{-}\right)\right)\right. \\
& :=\sum_{i=1}^{7} F_{i}(t) .
\end{aligned}
$$

We divide the proof into three steps.

Step 1 . We claim that $\pi$ is mean square continuous on $[0, \infty)$. Let $x \in H, t_{1} \geq 0$, and $|h|$ be sufficiently small, then

$$
\begin{aligned}
E\left|(\pi x)\left(t_{1}+h\right)-(\pi x)\left(t_{1}\right)\right|^{2} & \leq 7 \sum_{i=1}^{7} E\left|F_{i}\left(t_{1}+h\right)-F_{i}\left(t_{1}\right)\right|^{2} \\
& :=7 \sum_{i=1}^{7} E\left|\triangle F_{i}\left(t_{1}\right)\right|^{2} .
\end{aligned}
$$

We can easily see that $E\left|\triangle F_{i}\left(t_{1}\right)\right|^{2} \rightarrow 0, i=1,2,3,4,7$, as $h \rightarrow 0$. Moreover, by the properties of the martingales $[5,8]$, we have

$$
\begin{aligned}
E \mid \triangle & \left.F_{5}\left(t_{1}\right)\right|^{2} \\
\leq & 2\left(\int_{0}^{t_{1}}\left(E\left|\left(S\left(t_{1}+h-s\right)-S\left(t_{1}-s\right)\right) g(s, x(s-\delta(s)))\right|^{2}\right) d s\right) \\
& +2\left(\int_{t_{1}}^{t_{1}+h}\left(E\left|S\left(t_{1}+h-s\right) g(s, x(s-\delta(s)))\right|^{2}\right) d s\right) \\
\rightarrow & 0 \quad \text { as } h \rightarrow 0 . \\
E \mid \triangle & \left.F_{6}\left(t_{1}\right)\right|^{2} \\
\leq & 2 \int_{0}^{t_{1}} \int_{\mathcal{Z}} E\left|\left(S\left(t_{1}+h-s\right)-S\left(t_{1}-s\right)\right) h(s, x(s-\kappa(s), v))\right|^{2} \lambda(d v) d s \\
& +2 \int_{t_{1}}^{t_{1}+h} \int_{\mathcal{Z}} E\left|\left(S\left(t_{1}+h-s\right)-S\left(t_{1}-s\right)\right) h(s, x(s-\kappa(s), v))\right|^{2} \lambda(d v) d s \\
\rightarrow & 0 \quad \text { as } h \rightarrow 0 .
\end{aligned}
$$

Consequently, $\pi$ is mean square continuous on $[0, \infty)$.

Step 2. We claim that $\pi(H) \subset H$. From (4), we have

$$
\begin{aligned}
E|(\pi x)(t)|^{2} \leq & 7 E|S(t)[\varphi(0)-u(0, x(-\rho(0)))]|^{2}+7 E|u(t, x(t-\rho(t)))|^{2} \\
& +7 E\left|\int_{0}^{t} A S(t-s) u(s, x(s-\rho(s))) d s\right|^{2} \\
& +7 E\left|\int_{0}^{t} S(t-s) f(s, x(s-\tau(s))) d s\right|^{2} \\
& +7 E\left|\int_{0}^{t} S(t-s) g(s, x(s-\delta(s))) d w(s)\right|^{2}
\end{aligned}
$$




$$
\begin{aligned}
& +7 E \mid \int_{0}^{t} \int_{\mathcal{Z}} S(t-s) h\left(s,\left.x(s-\kappa(s), v) \tilde{N}(d s, d v)\right|^{2}\right. \\
& +7 E\left|\sum_{0<t_{k}<t} S\left(t-t_{k}\right) I_{k}\left(x\left(t_{k}^{-}\right)\right)\right|^{2}:=\sum_{i=1}^{7} G_{i}(t) .
\end{aligned}
$$

Note that $x(t) \in H$. By (H1), (H3), (H4) and Lemma 1, we have

$$
\begin{aligned}
& G_{1}(t) \leq 7 M^{2} e^{-2 a t}\left(1+\sqrt{\bar{K}}\left|(-A)^{-\alpha}\right|\right)^{2}\|\varphi\|_{C}^{2} \rightarrow 0 \quad \text { as } t \rightarrow \infty, \\
& G_{2}(t) \leq 7 \bar{K}\left|(-A)^{-\alpha}\right|^{2} E|x(t-\rho(t))|^{2} \rightarrow 0 \text { as } t \rightarrow \infty, \\
& G_{7}(t) \leq 7 M^{2} e^{-2 a t} q_{k} E\left|x\left(t_{k}^{-}\right)\right|^{2} \rightarrow 0 \text { as } t \rightarrow \infty .
\end{aligned}
$$

By Lemma 1, (H3) and the Hölder inequality, we obtain

$$
\begin{aligned}
G_{3}(t) & \leq 7 E\left(\int_{0}^{t}\left|(-A)^{-\alpha} S(t-s)(-A)^{\alpha} u(s, x(s-\rho(s)))\right| d s\right)^{2} \\
& \leq 7 M_{1-\alpha}^{2} \bar{K}\left(\int_{0}^{t} e^{-a(t-s)}(t-s)^{2 \alpha-2} d s\right) \int_{0}^{t} e^{-a(t-s)} E|x(s-\rho(s))|^{2} d s \\
& \leq 7 M_{1-\alpha}^{2} \bar{K} a^{1-2 \alpha} \Gamma(2 \alpha-1) \int_{0}^{t} e^{-a(t-s)} E|x(s-\rho(s))|^{2} d s .
\end{aligned}
$$

For any $x(t) \in H$ and $\epsilon>0$, there exists $t_{1}>0$ such that $E|x(t-\rho(t))|^{2}<\epsilon$ for $t \geq t_{1}$. We thus obtain

$$
\begin{aligned}
G_{3}(t) \leq & 7 M_{1-\alpha}^{2} \bar{K} a^{1-2 \alpha} \Gamma(2 \alpha-1) \int_{0}^{t_{1}} e^{-a(t-s)} E|x(s-\rho(s))|^{2} d s \\
& +7 M_{1-\alpha}^{2} \bar{K} a^{-2 \alpha} \Gamma(2 \alpha-1) \epsilon .
\end{aligned}
$$

We can see $e^{-a t} \rightarrow 0$ as $t \rightarrow \infty$. By (3), there exists $t_{2} \geq t_{1}$ such that for any $t \geq t_{2}$ we obtain

$$
\begin{aligned}
& 7 M_{1-\alpha}^{2} \bar{K} a^{1-2 \alpha} \Gamma(2 \alpha-1) \int_{0}^{t_{1}} e^{-a(t-s)} E|x(s-\rho(s))|^{2} d s \\
& \leq \epsilon-7 M_{1-\alpha}^{2} \bar{K} a^{-2 \alpha} \Gamma(2 \alpha-1) \epsilon .
\end{aligned}
$$

This, together with (11), yields for any $t \geq t_{2}, G_{3}(t) \leq \epsilon$. That is,

$$
G_{3}(t) \rightarrow 0 \quad \text { as } t \rightarrow \infty
$$

By $(\mathrm{H} 1),(\mathrm{H} 2)$, the Hölder inequality, Lemma 1 and the properties of the martingales $[5,8]$, we easily obtain

$$
\begin{aligned}
& G_{4}(t) \leq 7 M^{2} K a^{-1} \int_{0}^{t} e^{-a(t-s)} E|x(s-\tau(s))|^{2} d s \\
& G_{5}(t) \leq 7 M^{2} K \int_{0}^{t} e^{-2 a(t-s)} E|x(s-\delta(s))|^{2} d s .
\end{aligned}
$$




$$
\begin{aligned}
G_{6}(t) & \leq 7 \int_{0}^{t} \int_{\mathcal{Z}} E|S(t-s) h(s, x(s-\kappa(s), v))|^{2} \lambda(d v) d s \\
& \leq 7 M^{2} K \int_{0}^{t} e^{-2 a(t-s)} E|x(s-\kappa(s))|^{2} d s .
\end{aligned}
$$

Further, similar to the proof of (12), from (13), (14) and (15), we then have $G_{4}(t), G_{5}(t)$, $G_{6}(t) \rightarrow 0$ as $t \rightarrow \infty$. Therefore, we have $E|(\pi x)(t)|^{2} \rightarrow 0$ as $t \rightarrow \infty$. That is, $\pi(H) \subset H$.

Step 3 . We claim that $\pi$ is a contraction mapping. Let $x, y \in H$, we have

$$
\begin{aligned}
& \sup _{t \in[0, T]} E|(\pi x)(t)-(\pi y)(t)|^{2} \\
& \leq 6\left[\sup _{t \in[0, T]} E|u(t, x(t-\rho(t)))-u(t, y(t-\rho(t)))|^{2}\right. \\
&+\sup _{t \in[0, T]} E\left|\int_{0}^{t} A S(t-s)[u(s, x(s-\rho(s)))-u(s, y(s-\rho(s)))] d s\right|^{2} \\
&+\sup _{t \in[0, T]} E\left|\int_{0}^{t} S(t-s)[f(s, x(s-\tau(s)))-f(s, y(s-\tau(s)))] d s\right|^{2} \\
&+\sup _{t \in[0, T]} E\left|\int_{0}^{t} S(t-s)[g(s, x(s-\delta(s)))-g(s, y(s-\delta(t)))] d w(s)\right|^{2} \\
&+\sup _{t \in[0, T]} E\left|\int_{0}^{t} \int_{\mathcal{Z}} S(t-s)[h(s, x(s-\kappa(s), v))-h(s, y(s-\kappa(s), v))] \tilde{N}(d s, d v)\right|^{2} \\
&\left.+\sup _{t \in[0, T]} E\left|\sum_{0<t_{k}<t} S\left(t-t_{k}\right)\left[I_{k}\left(x\left(t_{k}^{-}\right)\right)-I_{k}\left(y\left(t_{k}^{-}\right)\right)\right]\right|^{2}\right] \\
& \leq 6\left[\bar{K}\left|(-A)^{-\alpha}\right|^{2}+\bar{K} M_{1-\alpha}^{2} a^{-2 \alpha} \Gamma(2 \alpha-1)+M^{2} K a^{-2}+K M^{2} a^{-1}\right. \\
&\left.+M^{2} \bar{L}\right] \sup _{t \in[0, T]} E|x(t)-y(t)|^{2},
\end{aligned}
$$

where $\bar{L}=e^{-2 a T} E\left(\sum_{k=1}^{m}\left|q_{k}\right|\right)$. Thus $\pi$ is a contraction mapping. Hence there exists a unique fixed point $x(t)$ in $H$ which is the solution of Eq. (1) and $E|x(t)|^{2} \rightarrow 0$ as $t \rightarrow \infty$. The proof is complete.

Similarly, we can easily generalize the above result to global asymptotic stability in the $p$ th moment.

Theorem 2 If (H1)-(H4) hold for some $\alpha \in(1 / p, 1], p \geq 2$, and the inequality

$$
\begin{aligned}
& 6^{p-1}\left[\bar{K}^{p / 2}\left|(-A)^{-\alpha}\right|^{p}+\bar{K}^{p / 2} M_{1-\alpha}^{p} a^{-p \alpha}(\Gamma(1+p(\alpha-1) /(p-1)))^{p-1}+M^{p} K^{p / 2} a^{-p}\right. \\
& \left.\quad+c_{p} M^{p} K^{p / 2}(2 a(p-1) /(p-2))^{1-p / 2} / a+M^{p} \bar{L}\right]<1
\end{aligned}
$$

also holds, then Eq. (1) is globally asymptotically stable in the pth moment, where $\bar{L}=$ $e^{-a p T} E\left(\sum_{k=1}^{m}\left|q_{k}\right|^{p}\right)$ and $c_{p}=(p(p-1) / 2)^{p / 2}$.

Remark Without the impulsive and Poisson jumps, Eq. (1) reduces to a stochastic partial differential equation, which is investigated in [10]. If without Poisson jumps, then Eq. (1) 
reduces to impulsive stochastic neutral partial differential equations, which is studied in [14]. If without the neutral term and Poisson jumps, then Eq. (1) reduces to an impulsive stochastic partial differential equation, which is studied in [21]. In the sense, the results of this paper are generalized.

\section{Conclusion}

This paper discusses the globally asymptotic stability of the mild solutions to jumpdiffusions of neutral type with impulses by the fixed point theory. Globally asymptotic stability of the mild solutions to jump-diffusions of neutral type with impulses are derived. Some earlier results are generalized and improved.

Competing interests

The authors declare that they have no competing interests.

Authors' contributions

FJ carried out the study of asymptotic stability of this paper and drafted the manuscript. HY participated in the proof of the main result of the paper. XZ provided some constructive suggestions for the improvement of the paper. All authors read and approved the final manuscript.

\section{Author details}

${ }^{1}$ School of Statistics \& Mathematics, Zhongnan University of Economics and Law, Wuhan, 430073, China. ${ }^{2}$ School of Mathematics \& Computer Science, Wuhan Polytechnic University, Wuhan, 430023, China.

\section{Acknowledgements}

We wish to thank the referees for their valuable suggestions which have considerably improved the presentation of this article. The work is supported by the Fundamental Research Funds for the Central Universities, the National Natural Science Foundation of China under Grant 61304067 and the Natural Science Foundation of Hubei Province of China under Grant 2013CFB443.

Received: 24 December 2012 Accepted: 29 October 2013 Published: 25 Nov 2013

\section{References}

1. Caraballo, T: Asymptotic exponential stability of stochastic partial differential equations with delay. Stochastics 33 27-47 (1990)

2. Caraballo, T, Liu, K: Exponential stability of mild solutions of stochastic partial differential equations with delays Stoch. Anal. Appl. 17, 743-763 (1999)

3. Da Prato, G, Zabczyk, J: Stochastic Equations in Infinite Dimensions. Cambridge University Press, Cambridge (1992)

4. Liu, K: Stability of Infinite Dimensional Stochastic Differential Equations with Applications. Chapman and Hall, London (2006)

5. Luo, J, Liu, K: Stability of infinite dimensional stochastic evolution equations with memory and Markovian jumps. Stoch. Process. Appl. 118, 864-895 (2008)

6. Mao, X: Stochastic Differential Equations and Applications. Horwood, Chichester (1997)

7. Benchohra, M, Henderson, J, Ntouyas, S: Impulsive Differential Equations and Inclusions. Hindawi Publishing Corporation, New York (2006)

8. Hu, J, Liu, X: Existence results of impulsive partial neutral integro-differential inclusions with infinity delay. Nonlinear Anal. 71, e1132-e1138 (2009)

9. Svishchuk, AV, Kazmerchuk, YI: Stability of stochastic delay equations of Ito form with jumps and Markovian switchings, and their applications in finance. Theory Probab. Math. Stat. 64, 167-178 (2002)

10. Mahmudov, NI: Existence and uniqueness results for neutral SDEs in Hilbert spaces. Stoch. Anal. Appl. 24, 79-95 (2006)

11. Bao, J, Hou, Z: Existence of mild solutions to stochastic neutral partial functional differential equations with non-Lipschitz coefficients. Comput. Math. Appl. 59, 207-214 (2010)

12. Govindan, TE: Almost sure exponential stability for stochastic neutral partial functional differential equations. Stochastics 77, 139-154 (2005)

13. Luo, J: Fixed points and stability of neutral stochastic delay differential equations. J. Math. Anal. Appl. 334, 431-440 (2007)

14. Jiang, F, Shen, Y: Stability of impulsive stochastic neutral partial differential equations with infinite delays. Asian J. Control 14, 1706-1709 (2012)

15. Chen, $\mathrm{H}$ : Integral inequality and exponential stability for neutral stochastic partial differential equations with delays. J. Inequal. Appl. 2009, 1-15 (2009)

16. Sakthivel, R, Luo, J: Asymptotic stability of impulsive stochastic partial differential equations with infinite delays. J. Math. Anal. Appl. 356, 1-6 (2009)

17. Bao, J, Truman, A, Yuan, C: Almost sure asymptotic of stochastic partial differential equations with jumps. SIAM J. Control Optim. 49, 771-787 (2011)

18. Bao, J, Truman, A, Yuan, C: Stability in distribution of mild solutions to stochastic partial differential equations with jumps. Proc. R. Soc. Lond., Ser. A, Math. Phys. Eng. Sci. 465, 2111-2134 (2009) 
19. Cui, J, Yan, L, Sun, X: Exponential stability for neutral stochastic partial differential equations with delays and Poisson jumps. Stat. Probab. Lett. 81, 1970-1977 (2011)

20. Peszat, S, Zabczyk, J: Stochastic Partial Differential Equations with Lévy Noise: An Evolution Equation Approach. Cambridge University Press, Cambridge (2007)

21. Sakthivel, R, Luo, J: Asymptotic stability of nonlinear impulsive stochastic differential equations. Stat. Probab. Lett. 79, 1219-1223 (2009)

22. Pazy, A: Semigroups of Linear Operators and Applications to Partial Differential Equations. Springer, New York (1992)

10.1186/1029-242X-2013-561

Cite this article as: Jiang et al.: On the asymptotic stability of a class of jump-diffusions of neutral type with impulses. Journal of Inequalities and Applications 2013, 2013:561

Submit your manuscript to a SpringerOpen ${ }^{\circ}$ journal and benefit from:

- Convenient online submission

- Rigorous peer review

- Immediate publication on acceptance

- Open access: articles freely available online

- High visibility within the field

- Retaining the copyright to your article

Submit your next manuscript at $>$ springeropen.com 